\title{
Safety and efficacy of silodosin for the treatment of benign prostatic hyperplasia
}

This article was published in the following Dove Press journal:

Clinical Interventions in Aging

21 June 20II

Number of times this article has been viewed

\author{
Masaki Yoshida' \\ Junzo Kudoh ${ }^{2}$ \\ Yukio Homma ${ }^{3}$ \\ Kazuki Kawabe ${ }^{4}$ \\ 'Department of Medical Informatics, \\ 2Department of Urology, Japan Labor \\ Health and Welfare Organization, \\ Kumamoto Rosai Hospital, Kumamoto, \\ Japan; ${ }^{3}$ Department of Urology, \\ Graduate School of Medicine, The \\ University of Tokyo, Tokyo, Japan; \\ ${ }^{4}$ Tokyo Teishin Hospital, Tokyo, Japan
}

Correspondence: Masaki Yoshida Department of Medical Informatics, Japan Labor Health and Welfare Organization, Kumamoto Rosai Hospital 3-30-34-I 402 Suizenji, Kumamoto 862-0950, Japan

$\mathrm{Tel}+81963829215$

Fax +8I 963829215

Email akko-maki@umin.net

\begin{abstract}
Lower urinary tract symptoms (LUTS) associated with benign prostatic hyperplasia (BPH) are highly prevalent in older men. Medical therapy is the first-line treatment for LUTS associated with BPH. Mainstays in the treatment of male LUTS and clinical BPH are the $\alpha_{1}$-adrenergic receptor antagonists. Silodosin is a new $\alpha_{1}$-adrenergic receptor antagonist that is selective for the $\alpha_{1 \mathrm{~A}}$-adrenergic receptor. By antagonizing $\alpha_{1 \mathrm{~A}}$-adrenergic receptors in the prostate and urethra, silodosin causes smooth muscle relaxation in the lower urinary tract. Since silodosin has greater affinity for the $\alpha_{1 \mathrm{~A}}$-adrenergic receptor than for the $\alpha_{1 \mathrm{~B}}$-adrenergic receptor, it minimizes the propensity for blood pressure-related adverse effects caused by $\alpha_{1 \mathrm{~B}}$-adrenergic receptor blockade. In the clinical studies, patients receiving silodosin at a total daily dose of $8 \mathrm{mg}$ exhibited significant improvements in the International Prostate Symptom Score and maximum urinary flow rate compared with those receiving placebo. Silodosin showed early onset of efficacy for both voiding and storage symptoms. Furthermore, long-term safety of silodosin was also demonstrated. Retrograde or abnormal ejaculation was the most commonly reported adverse effect. The incidence of orthostatic hypotension was low. In conclusion, silodosin, a novel selective $\alpha_{1 \mathrm{~A}}$-adrenergic receptor antagonist, was effective in general and without obtrusive side effects. This review provides clear evidence in support of the clinical usefulness of silodosin in the treatment of LUTS associated with BPH.
\end{abstract}

Keywords: $\alpha_{1 \mathrm{~A}}$-adrenoceptor antagonist, silodosin, selective, benign prostatic hyperplasia, lower urinary tract symptoms

\section{Benign prostatic hyperplasia}

Benign prostatic hyperplasia (BPH) is a common progressive disease among men, with an incidence that is age-dependent. Histological BPH, which typically develops after the age of 40 years, ranges in prevalence from $>50 \%$ at 60 years to as high as $90 \%$ by 85 years of age. ${ }^{1-3}$ BPH contributes to, but is not the single cause of, bothersome lower urinary tract symptoms (LUTS) that may affect quality of life. The prevalence of troublesome symptoms increases with age, with symptoms typically occurring in men aged $\geq 50$ years. $^{3}$

Histologically, BPH is characterized by a progressive increase in the number of epithelial and stromal cells, that develops initially in the periurethral area of the prostate gland. ${ }^{1,4,5}$ This cellular proliferative process increases prostatic smooth muscle tone, resulting in urethral constriction. ${ }^{6}$ Benign prostatic enlargement may also result from the proliferation of epithelial and stromal cells, and may further contribute to constriction of the urethra, leading to bladder outlet obstruction. Benign prostatic enlargement and bladder outlet obstruction do not occur in all men with 
histopathological BPH/LUTS, and the presence of benign prostatic enlargement does not necessarily mean that bladder outlet obstruction will develop. ${ }^{5}$

Approximately $50 \%$ of patients with histological $\mathrm{BPH}$ report moderate to severe $\mathrm{LUTS}^{2}{ }^{2}$ consisting of storage and voiding symptoms., ${ }^{2,3}$ Commonly reported storage-related symptoms include urinary frequency, urgency, and nocturia. Voiding symptoms, typically attributable to urethral obstruction, consist of decreased and intermittent force of the urinary stream and the sensation of incomplete bladder emptying. ${ }^{1}$ Although bothersome LUTS may affect quality of life by altering normal daily activities and sleep patterns, mortality associated with BPH is rare. ${ }^{1,7}$ Although uncommon, serious complications of BPH may occur, including acute urinary retention, renal insufficiency, urinary tract infections, hematuria, bladder stones, and renal failure. ${ }^{6,8}$ These complications may be triggered or worsened by inadequate management of $\mathrm{BPH}$. The incidence of acute urinary retention in untreated patients ranges from $0.3 \%$ to $3.5 \%$ per year; the risk of developing other long-term complications is unclear. ${ }^{8}$

The management of patients with BPH includes nonpharmacological, pharmacological, and surgical options, with the choice of therapy typically depending on the presence and severity of symptoms. ${ }^{1,9}$ Watchful waiting is the preferred management strategy for patients with mild LUTS and those who do not perceive their symptoms to be particularly bothersome. Pharmacological treatments include $\alpha_{1}$-adrenergic receptor antagonists (or blockers) and $5 \alpha$-reductase inhibitors, which are recommended for use alone or in combination in patients with bothersome moderate to severe LUTS. Currently, adrenergic receptor antagonists are commonly used as the first-line treatments for LUTS associated with BPH. ${ }^{3,6}$

\section{$\alpha_{1}$-Adrenergic receptors}

Adrenergic receptors were originally divided into $\alpha$-adrenergic receptor and $\beta$-adrenergic receptor categories, ${ }^{8}$ but application of molecular biological methods has confirmed nine adrenergic receptor subtypes: $\alpha_{1 \mathrm{~A}}$ (formerly named $\left.\alpha_{1 \mathrm{C}}\right), \alpha_{1 \mathrm{~B}}, \alpha_{1 \mathrm{D}}, \alpha_{2 \mathrm{~A}}, \alpha_{2 \mathrm{~B}}, \alpha_{2 \mathrm{C}}, \beta_{1}, \beta_{2}$, and $\beta_{3}{ }^{10-12}$ All three $\alpha_{1}$-adrenergic receptor subtypes exist in a wide range of human tissues. ${ }^{13,14}$

In terms of male LUTS, $\alpha_{1}$-adrenergic receptor expression in the prostate, urethra, spinal cord, and bladder is important. Molecular and contraction studies in human prostate tissue demonstrate that the $\alpha_{1 \mathrm{~A}}$-adrenergic receptor subtype predominates $(70 \%-100 \%)$ in prostate stroma. ${ }^{15,16}$ Because baseline tone is present in prostate smooth muscle (due to its rich sympathetic innervation), blockade of prostate $\alpha_{1 \mathrm{~A}}$-adrenergic receptors results in relaxation of prostate smooth muscles. Hence, $\alpha_{1}$-adrenergic receptor blockade is capable of modifying the dynamic (prostate smooth muscle contraction) component in BPH. Another tissue important in LUTS is the urethra. To date, most studies show that all regions of the human urethra (including bladder neck and intraprostatic urethra) mainly contain $\alpha_{1 \mathrm{~A}}$-adrenergic receptors. ${ }^{17,18}$

$\alpha_{1}$-Adrenergic receptor antagonists also mediate vasodilation in the vasculature; therefore, one of the side effects of treating LUTS with $\alpha_{1}$-adrenergic receptor antagonists is hypotension. Although the main $\alpha_{1}$-adrenergic receptor subtype in the large vasculature is the $\alpha_{1 \mathrm{~B}}$-adrenergic receptors, $\alpha_{1 \mathrm{~A}}$-adrenergic receptors predominate in human splanchnic (mesenteric, splenic, hepatic, and distal omental) resistance arteries. ${ }^{19}$ Interestingly, $\alpha_{1}$-adrenergic receptor expression increases two-fold in representative (mammary) arteries with aging, with the ratio of $\alpha_{1 \mathrm{~B}}: \alpha_{1 \mathrm{~A}}$ increasing, whereas no alteration occurs in veins. ${ }^{19}$ Studies of pharmacy databases in Europe suggest that the administration of $\alpha_{1}$-adrenergic receptor blockers increases the incidence of hip fractures (chosen as a surrogate for clinically important orthostatic hypotension). ${ }^{20}$ Further analysis with regard to the precise $\alpha_{1}$-adrenergic receptor antagonists prescribed suggests that avoidance of $\alpha_{1 \mathrm{~B}}$-adrenergic receptor blockade may result in fewer overall hip fractures. ${ }^{3}$

\section{Molecular and pharmacological characteristics of silodosin}

A number of $\alpha_{1}$-adrenergic receptor antagonists (alfuzosin, doxazosin, tamsulosin, terazosin, naftopidil) have been approved for the treatment of BPH worldwide. The earlier $\alpha_{1}$-adrenergic receptor antagonists cause vasodilatory symptoms, including postural hypotension and dizziness, and have to be used carefully in patients, especially in older patients suffering from dysuria. Tamsulosin has relative selectivity for the $\alpha_{1 \mathrm{~A}}$-adrenergic receptor. However, in patients with BPHrelated LUTS, it has long been desired to develop a therapeutic agent having a selective suppressive action on urethral contractions, with less hypotension, including postural hypotension. This effect may be minimized by use of agents that selectively antagonize the $\alpha_{1 \mathrm{~A}}$-adrenergic receptor. ${ }^{7}$ At the start of the 1990s, Kissei Pharmaceutical Co Ltd began development of $\alpha_{1}$-adrenergic receptor antagonists that were highly selective for the lower urinary tract without affecting blood pressure, ${ }^{21,22}$ and this led to the discovery of silodosin, a novel indoline derivative. 
Receptor-binding studies (saturation and replacement experiments) were performed using membrane fractions prepared from mouse-derived LM (tk-) cells expressing human $\alpha_{1 A^{-}}, \alpha_{1 B^{-}}$, or $\alpha_{1 D^{-}}$-adrenergic receptor and ${ }^{3} H$-prazosin hydrochloride, to study the affinity of silodosin for human $\alpha_{1}$-adrenergic receptor subtypes. As indicated in Table $1,{ }^{23}$ the affinity of silodosin for the $\alpha_{1 \mathrm{~A}}$-adrenergic receptor was 162 times higher than that for the $\alpha_{1 \mathrm{~B}}$-adrenergic receptor, and was 55 times higher than that for the $\alpha_{1 D}$-adrenergic receptor (calculated as a ratio of 162/2.95), having the highest selectivity for the $\alpha_{1 \mathrm{~A}}$-adrenergic receptor among the tested $\alpha_{1}$-adrenergic receptor antagonists.

The study was designed to determine the native tissue selectivity and $\alpha_{1}$-adrenoceptor subtype selectivity of silodosin by performing functional studies on contraction of isolated muscular preparations from the rabbit and the rat. Tissue samples of $\alpha_{1 \mathrm{~A}}$-adrenergic receptor-rich prostate, urethra, and bladder trigone isolated from male Japanese white rabbits, $\alpha_{1 \mathrm{~B}}$-adrenergic receptor-rich spleen isolated from male Sprague Dawley rats, and $\alpha_{1 D}$-adrenergic receptor-rich thoracic aorta also isolated from male Sprague Dawley rats were used to study the suppression of noradrenaline-induced contraction in a muscle bath by silodosin, tamsulosin hydrochloride, naftopidil, and prazosin hydrochloride. All of the tested $\alpha_{1}$-adrenergic receptor antagonists shifted the noradrenaline dose-response curves for the rabbit prostate, rat spleen, and rat thoracic aorta to the right in a concentration-dependent manner. ${ }^{23,24}$ The antagonistic action of silodosin against noradrenalineinduced contraction of each isolated tissue was compared with that of $\alpha_{1}$-adrenergic receptor antagonists by the $\mathrm{pA}_{2}$ or $\mathrm{pK}_{\mathrm{b}}$ value (Table 2). ${ }^{23}$ Silodosin was about 280 times more selective for prostate tissue than for splenic tissue and about 50 times more selective than for thoracic aortic tissue, which shows that silodosin is significantly more selective for prostate tissue compared with other $\alpha_{1}$-adrenergic receptor antagonists. Furthermore, the selectivity for the urethra and bladder trigone was found to be comparable with that for the prostate. The selectivity of tamsulosin hydrochloride for the prostate was about 20 times higher than that of selectivity for spleen, but comparable with that for the thoracic aorta. On the other hand, naftopidil and prazosin hydrochloride were more selective for the spleen and thoracic aorta $(0.4$ and 5 times for naftopidil and 25 and 20 times for prazosin hydrochloride, respectively), showing the selectivity for the prostate to be lower.

To evaluate in vivo uroselectivity (ratio of reactivities for lower urinary tract against blood pressure), several studies ${ }^{25,26}$ were performed, using rats. Intravenous dosing of phenylephrine, an $\alpha_{1}$-adrenergic receptor agonist, through the femoral vein increases intraurethral pressure in urethane-anesthetized male Sprague-Dawley rats. This effect should be blocked by $\alpha_{1}$-adrenergic receptor antagonists (Table 3 ). ${ }^{25}$ The results showed that each of the $\alpha_{1}$-adrenergic receptor antagonists dose-dependently suppressed the phenylephrine-induced increase in intraurethral pressure, lowering the mean blood pressure. Silodosin potently suppressed the phenylephrineinduced increase in intraurethral pressure, but tamsulosin hydrochloride equally suppressed the phenylephrine-induced increase in intraurethral pressure and also decreased the mean blood pressure at a similar dose. Naftopidil and prazosin hydrochloride showed a greater ability to decrease mean blood pressure in contrast with silodosin. Heart rate was decreased by about $10 \%$ by naftopidil at doses of $1000 \mu \mathrm{g} /$ $\mathrm{kg}$ and $3000 \mu \mathrm{g} / \mathrm{kg}$. No other antagonist had this effect. Efficacy in suppressing the phenylephrine-induced intraurethral pressure increase, defined by the $\mathrm{ID}_{50}$ value, was decreased by tamsulosin hydrochloride, silodosin, prazosin hydrochloride, and naftopidil (in descending order), and efficacy in decreasing mean blood pressure, defined by the $\mathrm{ED}_{15}$ value, decreased in order of prazosin hydrochloride, tamsulosin hydrochloride, silodosin, and naftopidil, showing that

Table I Affinity and selectivity for human $\alpha_{1}$-AR subtype for silodosin and other $\alpha_{1}-A R$ antagonists

\begin{tabular}{|c|c|c|c|c|c|}
\hline \multirow[t]{2}{*}{ Compound } & \multicolumn{3}{|c|}{$\mathrm{K}_{\mathrm{i}}$ value $(\mathrm{nmol} / \mathrm{L})$} & \multicolumn{2}{|c|}{$\alpha_{1}$-AR subtype selectivity ${ }^{a}$} \\
\hline & $\alpha_{I A}$-AR & $\alpha_{1 B}-A R$ & $\alpha_{1 D^{-A R}}$ & $\alpha_{I A} / \alpha_{1 B}$ ratio & $\alpha_{1 \mathrm{D}} / \alpha_{1 \mathrm{~B}}$ ratio \\
\hline Silodosin & $0.039 \pm 0.006$ & $6.5 \pm 0.6$ & $2.2 \pm 0.1$ & 162 & 2.95 \\
\hline Tamsulosin hydrochloride & $0.012 \pm 0.002$ & $0.12 \pm 0.00$ & $0.030 \pm 0.005$ & 9.55 & 3.80 \\
\hline Naftopidil & $23 \pm 7$ & $7.8 \pm 0.0$ & $4.4 \pm 0.4$ & 0.372 & 1.78 \\
\hline Prazosin hydrochloride & $0.12 \pm 0.01$ & $0.028 \pm 0.002$ & $0.078 \pm 0.007$ & 0.204 & 0.316 \\
\hline WB4I0I hydrochloride & $0.17 \pm 0.01$ & $I . I \pm 0 . I$ & $0.22 \pm 0.04$ & 6.03 & 5.01 \\
\hline BMY7378 dihydrochloride & $75 \pm 21$ & $28 \pm 7$ & $0.43 \pm 0.06$ & 0.389 & 64.6 \\
\hline
\end{tabular}

Notes: The $K_{i}$ value in the table presents the mean \pm standard error of 3 experiments. The subtype selectivity $\left(\alpha_{1 \mathrm{~A}} / \alpha_{1 \mathrm{~B}}\right.$ and $\alpha_{1 \mathrm{D}} / \alpha_{\mathrm{IB}}$ ratios) was calculated from the ratio after converting the concentration, specifically, using IOM $\left[\mathrm{M}=\mathrm{pK}_{\mathrm{i}}\left(\alpha_{1 \mathrm{~A}}\right.\right.$ or $\left.\left.\alpha_{\mathrm{ID}}\right)-\mathrm{pK}_{\mathrm{i}}\left(\alpha_{\mathrm{IB}}\right)\right]$.

Reproduced with permission from Yakugaku Zasshi Vol I26 Special issue. Copyright @ 2006 Pharmaceutical Society of Japan. ${ }^{23}$ 
Table $2 \mathrm{pA}_{2}$ or $\mathrm{pK}_{\mathrm{b}}$ values of silodosin and other $\alpha_{1}-\mathrm{AR}$ antagonists for noradrenaline-induced contraction in the isolated rabbit prostate, urethra and bladder, and in the isolated rat spleen and thoracic aorta

\begin{tabular}{|c|c|c|c|c|c|}
\hline \multirow[t]{3}{*}{ Compound } & \multicolumn{5}{|c|}{$\mathrm{pA}_{2}$ or $\mathrm{pK}_{\mathrm{b}}$ values } \\
\hline & \multicolumn{3}{|l|}{$\alpha_{1 A}$-AR } & \multirow{2}{*}{$\frac{\alpha_{1 B}-A R}{\text { Spleen }^{b}}$} & \multirow{2}{*}{$\frac{\alpha_{1 \mathrm{D}}-\mathrm{AR}}{\text { Thoracic aorta }^{\mathrm{b}}}$} \\
\hline & Prostate $^{a}$ & Urethra $^{a}$ & Bladder trigone $^{a}$ & & \\
\hline \multirow[t]{2}{*}{ Silodosin } & \multirow[t]{2}{*}{$9.60 \pm 0.05^{c}$} & $8.71 \pm 0.09$ & \multirow[t]{2}{*}{$9.35 \pm 0.15^{c}$} & $7.15 \pm 0.05$ & $7.88 \pm 0.05$ \\
\hline & & $(0.98 \pm 0.32)$ & & $(0.67 \pm 0.09 *)$ & $(1.00 \pm 0.18)$ \\
\hline Tamsulosin & \multirow[t]{2}{*}{$9.93 \pm 0.07^{c}$} & $9.00 \pm 0.06$ & \multirow[t]{2}{*}{$9.48 \pm 0.14^{c}$} & $8.64 \pm 0.06$ & $9.82 \pm 0.06$ \\
\hline hydrochloride & & $(1.16 \pm 0.27)$ & & $\left(0.61 \pm 0.18^{*}\right)$ & $(0.91 \pm 0.20)$ \\
\hline \multirow[t]{2}{*}{ Naftopidil } & $6.69 \pm 0.05^{c}$ & $6.48 \pm 0.11$ & $6.80 \pm 0.07$ & $6.30 \pm 0.07$ & $7.48 \pm 0.06$ \\
\hline & $(1.13 \pm 0.21)$ & $(0.93 \pm 0.39)$ & $(0.91 \pm 0.25)$ & $(0.69 \pm 0.23)$ & $(1.14 \pm 0.23)$ \\
\hline Prazosin & $7.91 \pm 0.02$ & $7.96 \pm 0.04$ & $8.10 \pm 0.05$ & $9.34 \pm 0.13$ & $9.17 \pm 0.06$ \\
\hline hydrochloride & $(1.08 \pm 0.09)$ & $\left(0.85 \pm 0.13^{*}\right)$ & $(0.97 \pm 0.18)$ & $\left(0.56 \pm 0.26^{*}\right)$ & $(1.11 \pm 0.23)$ \\
\hline \multicolumn{6}{|c|}{$\begin{array}{l}\text { Notes: Each value in the table presents mean } \pm \text { standard error of } 4-5 \text { animals. Each value in a parenthesis presents the slop of the Schild plot. a) Rabbits, b) Rats, c) pK; }{ }^{b} \text { value. } \\
\text { *Significantly different from the unity at } P<0.05 \text { by paired-t test. }\end{array}$} \\
\hline \multicolumn{6}{|c|}{$\begin{array}{l}\text { Abbreviations: } \mathrm{pA}_{2} \text {, negative logarithmic value of molar concentration of antagonistic blockers necessary to parallel shift } 2 \text { times the independent stimulant } \\
\text { concentration-reaction curve to the higher concentration side; } \mathrm{pK}_{\mathrm{b}} \text { value, negative logarithmic value of dissociation constant for binding of antagonistic blockers to } \\
\text { receptors. }\end{array}$} \\
\hline
\end{tabular}

silodosin $\left(\mathrm{ED}_{15} / \mathrm{ID}_{50}\right)$ has the highest selectivity for the lower urinary tract at 11.7 , followed by tamsulosin hydrochloride, prazosin hydrochloride, and naftopidil in this order. ${ }^{25}$

\section{Clinical efficacy and safety}

Four Phase III studies conducted in Japan, ${ }^{27}$ the US, ${ }^{28}$ and Europe $^{29}$ have evaluated the use of silodosin in the treatment of patients with BPH. The main efficacy results are summarized in Table 4.

The first randomized, double-blind, placebo-controlled Phase III study was conducted at 88 centers in Japan. ${ }^{27}$ The men included were aged $\geq 50$ years, were outpatients, and had LUTS associated with BPH, the latter diagnosed on a digital rectal examination or ultrasonographic findings. Inclusion criteria were a total International Prostate Symptom Score (IPSS) $\geq 8$, an associated quality of life score of $\geq 3$, prostate volume (measured by transabdominal or transrectal

Table $3 I_{50}$ value, ED ${ }_{15}$ value and uroselectivity of silodosin and other $\alpha_{1}$-AR antagonists after intravenous administration in the anesthetized rat

\begin{tabular}{llll}
\hline Drug & IUP & MBP & Uroselectivity \\
\cline { 2 - 4 } & ID $_{50}(\mu \mathrm{g} / \mathbf{k g})$ & $\mathrm{ED}_{15}(\mu \mathrm{g} / \mathbf{k g})$ & $\left.\mathbf{( E D}_{15} / \mathrm{ID}_{50}\right)$ \\
\hline Silodosin & 0.932 & 10.9 & 11.7 \\
Tamsulosin & 0.400 & 0.895 & 2.24 \\
hydrochloride & & & \\
Naftopidil & 361 & 48.1 & 0.133 \\
Prazosin & 4.04 & 0.792 & 0.196 \\
hydrochloride & & & \\
\hline
\end{tabular}

Notes: $I_{50}$ value (the dose to suppress IUP increase by $50 \%$ ); $E D_{15}$ value (the dose to decrease the MBP by 15\%).

Reproduced with permission from Yakugaku Zasshi Vol I 26 Special issue. Copyright (c) 2006 Pharmaceutical Society of Japan. ${ }^{25}$ ultrasonography) $\geq 20 \mathrm{~mL}$, a maximum urinary flow rate $\left(\mathrm{Q}_{\max }\right)<15 \mathrm{~mL} / \mathrm{sec}$ with a voided volume $\geq 100 \mathrm{~mL}$, and a residual urine volume $<100 \mathrm{~mL}$.

After completing a seven-day "washout" and a seven-day, single-blind, placebo runin period, patients were randomized to receive oral silodosin $4 \mathrm{mg}$ twice daily, tamsulosin $0.2 \mathrm{mg} /$ day, or placebo twice daily for 12 weeks. This study was performed as a double-dummy design. Drugs were prescribed as follows: silodosin group (silodosin $4 \mathrm{mg}$ twice a day, tamsulosin placebo twice a day); tamsulosin group (tamsulosin $0.2 \mathrm{mg}$ once a day in the morning, tamsulosin placebo once a day, silodosin placebo twice a day), and placebo group (silodosin placebo twice a day, tamsulosin placebo twice a day).

At the end of the washout period and at weeks 1, 2, 4, 8 and 12 during the treatment period, subjective symptoms (IPSS and quality of life scores) and medication compliance were recorded, and uroflowmetry and physical examination (blood pressure and heart rate) conducted. Clinical laboratory tests were conducted at the start of the observation period and at four and 12 weeks of treatment. All adverse events were recorded and assessed for severity and causal relationship with the investigational product.

The primary endpoint for evaluation of efficacy was change in total IPSS from baseline; secondary endpoints were change in $\mathrm{Q}_{\max }$ and evaluation of subjective symptoms, eg, IPSS voiding and storage scores and quality of life score.

In total, 457 patients were enrolled and randomized to receive silodosin $(n=176)$, tamsulosin $(n=192)$, or placebo $(n=89)$. One patient in the silodosin group was excluded from the full analysis set due to protocol violation. There 
Table 4 Results of pivotal Phase III clinical trials

\begin{tabular}{|c|c|c|c|c|c|}
\hline Study & Patients (n) & Baseline & Change & Baseline & Change \\
\hline & & \multicolumn{2}{|c|}{ IPSS, mean (SD) } & \multicolumn{2}{|c|}{$\mathbf{Q}_{\max }$, mean $(\mathrm{SD}), \mathrm{mL} / \mathrm{sec}$} \\
\hline \multicolumn{6}{|l|}{ Kawabe et $\mathrm{al}^{27}$} \\
\hline Silodosin 8 mg/day & 175 & I7.I (5.7) & $-8.3(6.4)^{*}$ & $9.88(2.75)$ & $1.70(3.31)$ \\
\hline Tamsulosin $0.2 \mathrm{mg} / \mathrm{day}$ & 192 & $17.0(5.7)$ & $-6.8(5.7)$ & $9.4 \mathrm{I}(2.8 \mathrm{I})$ & $2.60(3.98)$ \\
\hline Placebo & 89 & $17.1(6.1)$ & $-5.3(6.7)$ & $0.18(2.72)$ & $0.26(2.21)$ \\
\hline \multicolumn{6}{|c|}{ Marks et $\mathrm{a}^{28}$ (Pooled US studies) } \\
\hline Silodosin 8 mg/day & 466 & $21.3(5.1)$ & $-6.4(6.63)^{*}$ & $8.7(2.60)$ & $2.6(4.43)^{*}$ \\
\hline Placebo & 457 & $21.3(4.9)$ & $-3.5(5.84)$ & $8.9(2.80)$ & $1.5(4.36)$ \\
\hline Chapple et $\mathrm{al}^{29}$ & (ITT population) & & & & \\
\hline Silodosin 8 mg/day & 371 & $19(4)$ & $-7.0:-2.3[-3.2,-1.4]^{*}$ & $10.78(n=38 I)$ & 3.77 \\
\hline Tamsulosin $0.4 \mathrm{mg} / \mathrm{day}$ & 376 & $19(4)$ & $-6.7:-2.0[-2.9 .-1.1]^{*}$ & $10.27(n=384)$ & 3.53 \\
\hline \multirow[t]{2}{*}{ Placebo } & 185 & $19(4)$ & -4.7 & $10.32(n=190)$ & 2.93 \\
\hline & & \multicolumn{2}{|c|}{ IPSS voiding symptoms, mean (SD) } & \multicolumn{2}{|c|}{ IPSS storage symptoms, mean (SD) } \\
\hline \multicolumn{6}{|l|}{ Kawabe et $\mathrm{al}^{27}$} \\
\hline Silodosin 8 mg/day & 175 & $10.8(4.1)$ & $-5.8(4.6)^{*}$ & $6.4(3.0)$ & $-2.5(2.9)^{*}$ \\
\hline Tamsulosin $0.2 \mathrm{mg} / \mathrm{day}$ & 192 & $10.8(4.2)$ & $-4.8(4.1)$ & $6.2(2.9)$ & $-2.1(2.6)$ \\
\hline Placebo & 89 & $10.9(4.4)$ & $-3.8(4.8)$ & $6.3(2.8)$ & $-1.5(2.6)$ \\
\hline \multicolumn{6}{|c|}{ Marks et a ${ }^{28}$ (Pooled US studies) } \\
\hline Silodosin 8 mg/day & 466 & $12(3.6)$ & $-4.0(4.3 I)^{*}$ & $9.3(2.6)$ & $-2.3(2.93)^{*}$ \\
\hline Placebo & 457 & $12(3.5)$ & $-2.1(3.76)$ & $9.3(2.5)$ & $-1.4(2.99)$ \\
\hline \multicolumn{6}{|l|}{ Chapple et $\mathrm{al}^{29}$} \\
\hline Silodosin 8 mg/day & 381 & $11.3(3.13)$ & $-4.5:-1.7:[-2.2,-1.1]^{*}$ & $7.9(2.49)$ & $-2.5:-0.7[-1.1,-0.2]^{*}$ \\
\hline Tamsulosin $0.4 \mathrm{mg} / \mathrm{day}$ & 384 & $11.0(3.27)$ & $-4.2:-1.4[-2.0,-0.8]^{*}$ & $7.9(2.5 \mathrm{I})$ & $-2.4:-0.6[-1.1,-0.2]^{*}$ \\
\hline Placebo & 190 & I I.3 (3.22) & -4.7 & $8.0(2.64)$ & -1.8 \\
\hline
\end{tabular}

Note: *Significant difference versus placebo.

Abbreviations: IPSS, International Prostate Symptom Score; $\mathrm{Q}_{\max }$, maximum flow rate; SD, standard deviation; ITT, intention to treat; [95\% Cl: confidence interval]

were no significant differences among the three groups in baseline characteristics, except for the quality of life score. Therefore, an adjusted analysis by baseline quality of life score was used for the primary endpoint.

The primary outcome measure, ie, change in total IPSS from baseline, was $-8.3 \pm 6.4,-6.8 \pm 5.7$, and $-5.3 \pm 6.7$ in the silodosin, tamsulosin, and placebo groups, respectively. There were significantly greater decreases with silodosin than placebo from one week after starting treatment. In the early-stage comparison, silodosin elicited a significantly larger decrease in IPSS than did tamsulosin at two weeks. The mean $(95 \%$ confidence interval $[\mathrm{CI}]$ ) intergroup differences in the total IPSS between silodosin and placebo, and between silodosin and tamsulosin, were $-3.0(-4.6,-1.3)$ and $-1.4(-2.7,-0.2)$, respectively, thus confirming that silodosin was better than placebo and not inferior to tamsulosin (both $P<0.001$ ). Figure 1 shows the time course of change in total IPSS score in this randomized, placebo-controlled, double-blind Phase III study. ${ }^{27}$

Silodosin was significantly better than placebo in terms of quality of life score $(P<0.002)$. Voiding symptoms (as measured by components of the total IPSS) were significantly improved in the silodosin group compared with the tamsulosin and placebo groups. ${ }^{21}$ The mean \pm standard deviation [SD] changes from baseline in IPSS subscores were $-5.8 \pm 4.6,-4.8 \pm 4.1$, and $-3.8 \pm 4.8$ in the silodosin, tamsulosin, and placebo groups, respectively $(P=0.023$, silodosin versus tamsulosin; $P<0.001$, silodosin versus placebo). Mean changes from baseline in storage symptoms were $-2.5 \pm 2.9,-2.1 \pm 2.6$, and $-1.5 \pm 2.6$ in the respective groups $(P<0.006$, silodosin versus placebo; silodosin versus

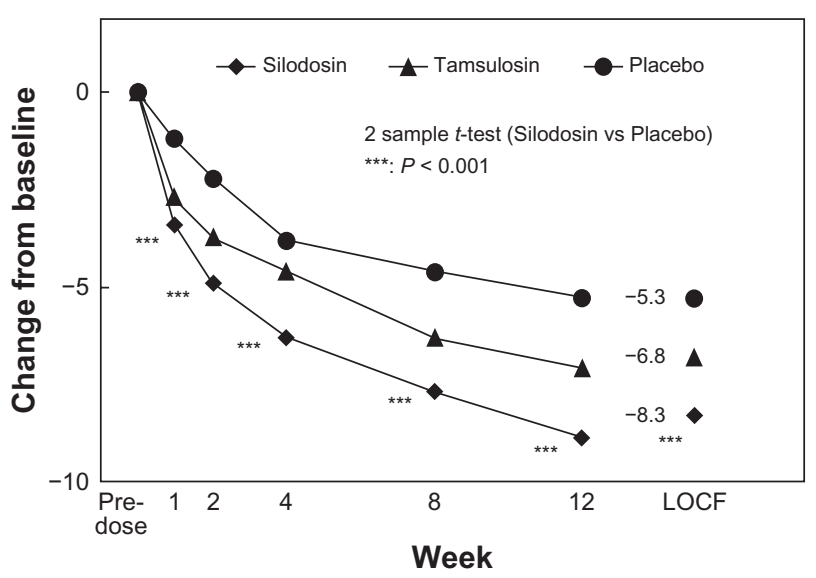

Figure I Time course of change in International Prostate Symptom Score in randomized, placebo-controlled, double-blind Phase III study.

Copyright (c) 2006, John Wiley and Sons. Reproduced with permissions from Kawabe et al. ${ }^{27}$ 
tamsulosin, not significant). In addition to significant effects in patients with moderate symptoms (IPSS 8-19), silodosin also showed significant improvements in total IPSS over placebo in patients with severe symptoms (IPSS $\geq 20$ ).

All three groups had improvements from baseline in $\mathrm{Q}_{\max }$ at week 12, and there were no significant differences in the change in $\mathrm{Q}_{\max }$ between groups. ${ }^{21}$ It is known that $\mathrm{Q}_{\max }$ depends on the voided volume at measurement. Therefore, the change in $\mathrm{Q}_{\max }$ was compared among the three treatment groups in the subgroup of patients with a change in voided volume of $<50 \%$ before and after treatment. In this analysis, the improvement in $\mathrm{Q}_{\max }$ from baseline was significantly greater in the silodosin group compared with the placebo group $(P=0.005)$, with mean $\pm \mathrm{SD}$ changes from baseline of $1.70 \pm 3.31,2.60 \pm 3.98$, and $0.26 \pm 2.21 \mathrm{~mL} / \mathrm{sec}$ in the silodosin, tamsulosin, and placebo groups, respectively.

The incidence of adverse events was $88.6 \%, 82.3 \%$, and $71.6 \%$ in the silodosin, tamsulosin, and placebo groups, respectively. Intergroup comparisons showed that adverse events were significantly $(P<0.001)$ more frequent in the silodosin group than in the placebo group. The incidence rates of drugrelated adverse events were $69.7 \%, 47.4 \%$, and $36.4 \%$ in the three groups, respectively, showing a significantly $(P<0.001)$ higher frequency of adverse events in the silodosin group than in the placebo and tamsulosin groups. Adverse events resulting in withdrawal occurred in $18(10.2 \%), 11(5.7 \%)$, and four $(4.5 \%)$ patients in the silodosin, tamsulosin, and placebo groups, respectively. All of these adverse events resolved after discontinuing treatment. The most common adverse event in the silodosin group was abnormal ejaculation, which occurred in 39 patients $(22.3 \%)$ compared with three $(1.6 \%)$ in the tamsulosin group and none in the placebo group. However, only five men (2.9\%) discontinued treatment due to abnormal ejaculation. Other adverse events occurring in the silodosin group at a frequency $>5 \%$ and more frequently than in the placebo group included upper respiratory tract infection $(18.9 \%$ silodosin, 27.6\% tamsulosin, and $0 \%$ placebo), thirst $(10.3 \%$, $3.6 \%$, and $4.5 \%$, respectively), loose stool ( $9.1 \%, 3.6 \%$, and $5.6 \%)$, diarrhea $(6.9 \%, 6.8 \%$, and $5.6 \%)$, urinary incontinence $(6.3 \%, 5.7 \%$, and $0 \%)$, and dizziness $(5.1 \%, 7.3 \%$, and $4.5 \%)$. There were no clinically significant differences in systolic/ diastolic blood pressure or heart rate between the silodosin and tamsulosin groups. In addition, the incidence of side effects relating to hypotension (such as dizziness) for silodosin was similar to that for tamsulosin and placebo.

The efficacy and safety of long-term administration of silodosin in patients with LUTS associated with BPH was investigated in a 52-week, open-label, oral administration study in 361 outpatients aged 50 years or older (mean age $67.3 \pm 6.7$ years) with a total IPSS score $\geq 8$, quality of life score $\geq 3$, prostate volume $\geq 20 \mathrm{~mL}$, voiding volume $\geq 100 \mathrm{~mL}$, and $\mathrm{Q}_{\max } \leq 15 \mathrm{~mL} / \mathrm{sec}$ at this time point. ${ }^{30}$ Silodosin $4 \mathrm{mg}$ (reduced to $2 \mathrm{mg}$ when an adverse event occurred) was administered twice daily after breakfast and dinner. This study was not an open-label extension of the pivotal Phase III study, but a stand-alone open-label study.

The study results showed that the total IPSS score was $18.4 \pm 6.3$ at baseline, $13.1 \pm 6.3$ at week $4,10.6 \pm 6.0$ at week $12,9.4 \pm 6.1$ at week 28 , and $8.2 \pm 5.7$ at week 52 , demonstrating a benefit over 52 weeks beginning at week 4 . For the IPSS subscores, the score for voiding symptoms was $10.9 \pm 4.5$ at baseline, $7.5 \pm 4.5$ at week 4 , and $4.4 \pm 3.9$ at week 52 , and for storage symptoms, $7.5 \pm 3.2$ at baseline, $5.6 \pm 2.9$ at week 4 , and $3.8 \pm 2.4$ at week 52 , demonstrating sustained improvement from as early as week $4(P=0.000)$. Additionally, the IPSS subscores for residual sensation, intermittency of urination, urinary stream, straining urination, pollakisuria, urinary urgency, and nocturia similarly lasted for 52 weeks from as early as week $4(P=0.000)$.

The quality of life score of all patients administered silodosin was $4.8 \pm 0.9$ at baseline, and $3.7 \pm 1.3$ at week 4 , $3.3 \pm 1.3$ at week $12,3.0 \pm 1.4$ at week 28 , and $2.7 \pm 1.3$ at week 52 , also showing that the improvement in quality of life lasted for 52 weeks from as early as week $4(P=0.000)$.

The $\mathrm{Q}_{\max }$ was $9.51 \pm 3.09 \mathrm{~mL} / \mathrm{sec}$ at baseline, $11.35 \pm 4.68 \mathrm{~mL} / \mathrm{sec}$ at week $4,10.57 \pm 4.68 \mathrm{~mL} / \mathrm{sec}$ at week $12,11.07 \pm 4.69 \mathrm{~mL} / \mathrm{sec}$ at week 28 , and $12.36 \pm 5.74 \mathrm{~mL} / \mathrm{sec}$ at week 52 , also showing improved efficacy lasting over 52 weeks and starting as early as week 4 . In addition, the residual urine volume was $44.5 \pm 61.1 \mathrm{~mL}$ at baseline and $30.2 \pm 39.2 \mathrm{~mL}$ at week 52, again showing improvement $(P=0.000)$.

Two US clinical studies that evaluated the efficacy and tolerability of silodosin $8 \mathrm{mg}$ once daily in men with BPH are described individually, and were pooled and reported by Marks et al. ${ }^{28}$ Both were 12 -week, multicenter, randomized, double-blind, placebo-controlled trials. The two studies enrolled patients aged $\geq 50$ years who had an IPSS $\geq 13$, a $Q_{\max } 4-15 \mathrm{~mL} / \mathrm{sec}$, and a postvoid residual volume $<250 \mathrm{~mL}$. The studies began with a four-week placebo runin period; patients with a $>30 \%$ decrease in IPSS or a $>3 \mathrm{~mL} / \mathrm{sec}$ increase in $\mathrm{Q}_{\max }$ at the end of this period were excluded from subsequent randomization. The enrolled men showed an average IPSS score of 21.2-21.4 points and a $\mathrm{Q}_{\max }$ of $8.4-9.0 \mathrm{~mL} / \mathrm{sec}$. After treatment, the IPSS improvements were 6.3 and 6.5 points versus 3.4 and 3.6 points in the placebo arms, respectively, and the flow 
rate improvements were 2.2 and $2.9 \mathrm{~mL} / \mathrm{sec}$ versus 1.2 and $1.9 \mathrm{~mL} / \mathrm{sec}$, respectively. Furthermore, the pooled data from the two trials were evaluated by Marks et al in their assessment of the efficacy and safety of silodosin for treatment of LUTS and BPH. Of 923 patients (mean age 65 years), 466 received silodosin and 457 received placebo. After 0.5 weeks (range 3-4 days) of treatment, patients receiving silodosin versus placebo achieved significant improvement in total IPSS (difference $-1.9, P<0.0001$ ), and storage $(-0.5$, $P<0.0002)$ and voiding $(-1.4, P<0.0001)$ subscores. The mean \pm SD change from baseline in total IPSS was $-4.2 \pm 5.3$ for silodosin versus $-2.3 \pm 4.4$ for placebo. Differences (silodosin versus placebo) in IPSS and subscores increased by week $12(P<0.0001)$. Mean change from baseline in $\mathrm{Q}_{\max } 2-6$ hours after the initial dose was greater $(P<0.0001)$ with silodosin $(2.8 \pm 3.4)$ than with placebo (1.5 \pm 3.8$)$. Differences remained significant $(P<0.001)$ through week 12 . The most common treatment-emergent adverse event was mild retrograde ejaculation (silodosin $28.1 \%$ of patients, placebo $0.9 \%$ ). Few patients treated with silodosin $(2.8 \%)$ discontinued because of retrograde ejaculation. Proportions of patients with treatment-emergent orthostatic hypotension were similar for silodosin $(2.6 \%)$ and placebo (1.5\%).

The report suggested that significant changes were observed at the earliest postbaseline assessments. IPSS, including storage and voiding subscores, improved significantly $(P<0.0005)$ within 3-4 days. Moreover, significant improvement $(P<0.0001)$ in $\mathrm{Q}_{\max }$ was observed 2-6 hours after the first dose of silodosin. Silodosin was safe and well tolerated. Retrograde ejaculation was the most common drugrelated adverse event but rarely resulted in discontinuation of treatment. In addition, silodosin had a low incidence of orthostatic hypotension, and was associated with few events of dizziness. The rapid onset of clinical efficacy established for silodosin would make it a useful option for the treatment of patients with signs and symptoms of BPH. A long-term open-label extension study of patients from these two US studies was also conducted over 40 weeks, with all patients receiving silodosin $8 \mathrm{mg}$ once daily. ${ }^{31}$

In Europe, a new multicenter double-blind, placebocontrolled and active-controlled parallel-group clinical study was conducted in 72 hospital clinics and inpatient units in 11 countries. ${ }^{29}$ After a washout phase of 14 days and a four-week, single-blind, placebo runin period, subjects who met the selection criteria were randomly assigned (in a ratio of $2: 2: 1$ ) to a 12 -week treatment with silodosin $8 \mathrm{mg}$, tamsulosin $0.4 \mathrm{mg}$, or placebo, administered once daily. Men aged 50 years and over with LUTS (defined by a stable total
IPSS score $\geq 13$ ), bladder outlet obstruction (defined by $\mathrm{Q}_{\max }$ 4-15 mL/sec and a minimum voided volume of $125 \mathrm{~mL}$ ).

A total of 1228 patients were screened, 955 of whom were randomized to receive silodosin $8 \mathrm{mg}(\mathrm{n}=381)$, tamsulosin $0.4 \mathrm{mg}(\mathrm{n}=384)$, or placebo $(\mathrm{n}=190)$. The study investigated whether silodosin was noninferior to tamsulosin and superior to placebo. The first endpoint was evaluation of IPSS; the secondary ones were urinary storage and voiding symptoms, quality of life, and $\mathrm{Q}_{\max }$. Treatment responders were defined as those having a $25 \%$ decrease in IPSS and a $30 \%$ increase in $\mathrm{Q}_{\max }$ from baseline. In the primary endpoints, superiority of silodosin and tamsulosin treatments versus placebo was observed, with highly statistically significant differences at all weeks $(P<0.001)$ both in the intention-to-treat (difference from placebo, -2.3 and -2.0 , respectively) and per protocol populations (difference from placebo, -2.2 and -1.9, respectively). In all three treatment groups, the percentage of IPSS responders progressively increased from baseline to week 12 . At study end, $66.8 \%$ and $65.4 \%$ of the patients receiving silodosin or tamsulosin, respectively, were responders compared with $50.8 \%$ in the placebo group. The differences versus placebo were highly significant $(P<0.001)$ for both active compounds, whereas the comparison between silodosin and tamsulosin did not show a statistically significant difference. The same results as previous studies were obtained from the analysis of urinary storage and voiding symptoms, when compared with placebo. Only for nocturia did silodosin have an advantage over tamsulosin, but this was not statistically significant $(P=0.095$ for tamsulosin and the placebo, $P=0.314$ for silodosin versus tamsulosin, and $P=0.013$ for silodosin versus placebo). However, there was no significant difference in $\mathrm{Q}_{\max }$ (responders $46.6 \%$ silodosin, $46.5 \%$ tamsulosin, and $40.5 \%$ placebo; responders had reduction $>30 \%$ from baseline) between the two active drugs and placebo. There was also no difference between the two drugs for the quality of life parameter, whereas both were better than for placebo. The adverse events for the three groups were $34.9 \%$ for silodosin, $28.9 \%$ for tamsulosin, and $24.2 \%$ for placebo, and disturbances in ejaculatory function were significantly greater in the group treated with silodosin $(14.2 \%)$ than in those treated with tamsulosin $(2.1 \%)$ or placebo (1.1\%). When analyzing cardiovascular adverse events, no statistically significant differences were found in laboratory parameters, vital signs, and electrocardiograms for silodosin and tamsulosin when compared with placebo. There were significant greater variations in blood pressure and heart rate for silodosin than for tamsulosin when compared with placebo. 
The urodynamic effects of silodosin were assessed in two Japanese studies employing invasive pressure flow measurements. Yamanishi et $\mathrm{a}^{32}$ treated 36 patients with LUTS and $\mathrm{BPH}$ and performed pressure flow studies at baseline and at three months, noting a decrease in detrusor pressure at maximal flow (pdet $\mathrm{Q}_{\max }$ ) from 80.6 to $48.6 \mathrm{~cm} \mathrm{H}_{2} \mathrm{O}$ and a decrease in the bladder outlet obstruction index from 70.2 to 32.6 ( $P<0.0001$ for both). In a similar study, Matsukawa et $\mathrm{al}^{33}$ treated 57 patients aged $51-84$ (mean 68.5) years with a prostate volume of $25-120 \mathrm{~mL}$ (mean $42.0 \mathrm{~mL}$ ) with silodosin $8 \mathrm{mg}$ for four weeks, and performed pressure flow studies before and after. Total IPSS and IPSS quality of life scores were significantly improved after drug administration. On free uroflowmetry, $\mathrm{Q}_{\max }$ and postvoid residual volume were significantly improved without a significant change in voided volume. Bladder volume at first desire to void significantly increased, but maximum cystometric capacity showed no significant change. Uninhibited detrusor contraction was observed in 24 patients $(42.1 \%)$ before silodosin administration. After drug administration, 14 of the 24 patients $(58.3 \%)$ had apparent improvement in detrusor overactivity, and uninhibited detrusor contraction disappeared in six patients $(25 \%)$. In eight patients $(33.3 \%)$ in whom uninhibited detrusor contraction amplitude showed a remarkable decrease of greater than $15 \mathrm{~cm} \mathrm{H}_{2} \mathrm{O}$, the mean amplitude of uninhibited detrusor contraction decreased from 51.6 to $11.5 \mathrm{~cm} \mathrm{H}_{2} \mathrm{O}$. None of the 33 patients who were free from uninhibited detrusor contraction before administration showed uninhibited contraction upon testing after administration. $\mathrm{Q}_{\max }$ and postvoid residual volume significantly improved after silodosin administration, similar to findings on free uroflowmetry. After administration, Pdet $\mathrm{Q}_{\max }$ significantly decreased from 72.5 to $51.4 \mathrm{~cm} \mathrm{H}_{2} \mathrm{O}$ for a mean decrease of $21.1 \mathrm{~cm} \mathrm{H_{2 }}$ O. Mean bladder outlet obstruction index significantly decreased from 60.6 to 33.8 after administration $(P<0.0001)$. These findings suggest the possibility that the highly selective effect on the $\alpha_{1 \mathrm{~A}}$ receptor at the bladder neck might be responsible for the observed reduction in obstruction, which is nearly commensurate with the effect of surgical intervention.

The efficacy and safety of silodosin and tamsulosin in patients with LUTS/BPH were also evaluated by a randomized crossover method. ${ }^{34} \mathrm{BPH}$ patients with IPSS $\geq 8$, quality of life score $\geq 3$, prostate volume $\geq 20 \mathrm{~mL}$, void volume $\geq 100 \mathrm{~mL}$, and $\mathrm{Q}_{\max }<15 \mathrm{~mL} / \mathrm{sec}$ were included, and were randomly divided into two groups, ie, a silodosin group (four weeks of twice-daily administration of silodosin $4 \mathrm{mg}$, followed by four weeks of once-daily administration of tamsulosin $0.2 \mathrm{mg}$ ) or a tamsulosin-preceding group (four weeks' administration of tamsulosin followed by four weeks' administration of silodosin). Forty-six patients were assigned to the silodosin-preceding group and 51 patients to the tamsulosin-preceding group. In the first treatment period, both drugs significantly improved the total IPSS score, but the improvement on silodosin was significantly superior to that on tamsulosin. After crossover treatment, significant improvement was observed only with silodosin treatment. Moreover, intergroup comparison of changes revealed that silodosin showed significant improvement of straining and nocturia with first and crossover treatments, respectively, compared with tamsulosin. Silodosin also significantly improved quality of life score in both treatment periods, while tamsulosin significantly improved quality of life score only in the first treatment period. Adverse drug reactions were observed in 16 of 97 patients (16.5\%) after administration of silodosin and in two of 97 patients $(2.1 \%)$ after administration of tamsulosin. The most frequent adverse drug reaction was ejaculatory disorder with silodosin in seven patients (7.2\%). However, the incidence of dizziness with silodosin was similar to that with tamsulosin. The authors concluded that silodosin exhibits excellent efficacy in improving subjective symptoms in both initial and crossover treatment, and it appears to improve the quality of life of patients with BPH/LUTS.

Table 5 Adverse effects of silodosin compared with tamsulosin and placebo

\begin{tabular}{|c|c|c|c|}
\hline Adverse effects & Silodosin (\%) & Tamsulosin (\%) & Placebo (\%) \\
\hline $\begin{array}{l}\text { Ejaculatory } \\
\text { disorders } \\
\text { (Retrograde } \\
\text { ejaculation) }\end{array}$ & $|4.2-28|$. & $1.6-2.1$ & $0-1.1$ \\
\hline $\begin{array}{l}\text { Upper respiratory } \\
\text { tract infection }\end{array}$ & 18.9 & 27.6 & 19.1 \\
\hline Thirst & 10.3 & 3.6 & 4.5 \\
\hline Loose stool & 9.1 & 3.6 & 5.6 \\
\hline $\begin{array}{l}\text { Urinary } \\
\text { incontinence }\end{array}$ & 6.3 & 5.7 & 0 \\
\hline Diarrhea & $2.6-6.9$ & 6.8 & 5.6 \\
\hline Dizziness & $3.2-5.1$ & 7.3 & 4.5 \\
\hline $\begin{array}{l}\text { Orthostatic } \\
\text { hypotension }\end{array}$ & 2.6 & - & 1.5 \\
\hline Headache & $2.4-5.5$ & 2.9 & $0.9-4.7$ \\
\hline $\begin{array}{l}\text { Discontinued the } \\
\text { study due to TEAE }\end{array}$ & $2.1-10.7$ & $1.0-5.7$ & $1.6-4.5$ \\
\hline $\begin{array}{l}\text { Discontinued the } \\
\text { study due to } \\
\text { ejaculatory } \\
\text { disorders }\end{array}$ & $1.3-2.9$ & 0.3 & 0 \\
\hline
\end{tabular}




\section{Safety profile}

Despite its high uroselectivity, silodosin is associated with side effects (summarized in Table 5). As described previously, the most commonly reported adverse reactions in the Phase III studies ${ }^{27-29}$ were ejaculatory disorders, including retrograde ejaculation $(22.3 \%, 28.1 \%$, and $14.2 \%$, respectively, compared with $1.6 \%$ and $2.1 \%$ with tamsulosin and $0 \%-1.1 \%$ with placebo). This adverse event was the main cause of discontinuation of silodosin $(2.8 \%, 2.9 \%$, and $1.3 \%$, respectively). The ejaculatory disorders are the result of smooth muscle relaxation in the prostate, urethra, bladder neck, and vas deferens. ${ }^{35,36}$ The $\alpha_{1 \mathrm{~A}}$-adrenergic receptor is mainly expressed in the bladder neck, vas deferens, and seminal vesicles. ${ }^{37}$ Thereby, this adverse reaction is explained by the high $\alpha_{1 \mathrm{~A}}$-adrenergic receptor subtype selectivity of silodosin. Relaxation of the prostate, urethra, and bladder neck might cause retrograde ejaculation.

In addition, nonclinical studies have shown that $\alpha$-adrenergic receptors, particularly $\alpha_{1 \mathrm{~A}}$-adrenergic receptors, are essential for the physiological contraction of the vas deferens and hence for sperm delivery from the testes to the urethra. ${ }^{38}$ Reduced ejaculation is caused by impaired function of the vas deferens rather than by alterations in sperm formation, number, or function. ${ }^{35}$ This effect does not represent a safety concern because it indicates only a reduction in semen volume, which is reversible (within a few days) upon discontinuation of treatment, ${ }^{36,39}$ and is not perceived as particularly bothersome (discontinuation rates due to ejaculation disorders in patients treated with silodosin were very low in the Phase III studies).

Silodosin for treatment of BPH symptoms was analyzed by two Phase III studies from the US to examine the relationship between treatment efficacy and occurrence of abnormal ejaculation. ${ }^{40}$ Silodosin-treated patients were stratified by the absence or presence of retrograde ejaculation. Groups were compared using analysis of covariance (for change from baseline) and responder analyses. Of 466 patients receiving silodosin, 131 (28\%) reported retrograde ejaculation and 335 (72\%) did not; four of the 457 patients receiving placebo $(0.9 \%)$ reported retrograde ejaculation. Most retrograde ejaculation events in silodosin-treated patients $(110 / 134$, $82 \%)$ were reported as "orgasm with absence of seminal emission". Silodosin-treated patients with and without retrograde ejaculation showed significant improvement in IPSS, $\mathrm{Q}_{\max }$, and quality of life versus placebo $(P<0.02)$. Patients with retrograde ejaculation versus patients without retrograde ejaculation experienced numerically greater improvement, but differences were not statistically significant
$(P>0.05)$. For patients with retrograde ejaculation, the odds of achieving improvement of $\geq 3$ in IPSS and $\geq 3 \mathrm{~mL} / \mathrm{sec}$ in $\mathrm{Q}_{\max }$ by study end were 1.75 times those for patients without retrograde ejaculation $(P=0.0127)$. Absence of seminal emission may predict superior treatment efficacy of silodosin in individual patients. A similar study was reported from Japan. ${ }^{41}$ The silodosin-treated group with ejaculation disorder (SIL + EjD) showed larger change in total IPSS than the silodosin subgroup without ejaculation disorder (SIL - EjD, difference -4.36 [95\% CI -6.44, -2.27]) and the placebo group (difference -6.29 [95\% CI -8.44, -4.14]). Remarkable improvement was observed at all time points The success rate in SIL + EjD was higher than in SIL - EjD and placebo when measured using a $25 \%$ reduction in the total IPSS category. There were no significant differences in adverse drug reaction rates other than for ejaculation disorder. Discontinuation rates between SIL + EjD and SIL - EjD were similar. The authors suggest that ejaculation disorder caused by selective $\alpha_{1 \mathrm{~A}}$-adrenergic receptor antagonists is associated with very large improvements in LUTS. Patients with ejaculation disorder may have greater symptomatic improvements without incremental risk for adverse events.

The other adverse events commonly associated with silodosin were upper respiratory tract infection $(18.9 \%$ versus $27.6 \%$ and $19.1 \%$ with tamsulosin and placebo, respectively), thirst $(10.3 \%$ versus $3.6 \%$ and $4.5 \%)$, loose stools $(9.1 \%$ versus $3.6 \%$ and $5.6 \%)$, urinary incontinence $(6.3 \%$ versus $5.7 \%$ and $0 \%$ ), diarrhea $(2.6 \%-6.9 \%$ versus $6.8 \%$ and $5.6 \%)$, dizziness $(3.2 \%-5.1 \%$ versus $7.3 \%$ and $4.5 \%$ ), and orthostatic hypotension ( $2.6 \%$ versus $1.5 \%$ for placebo). ${ }^{27-29}$

In a 52-week, long-term study in Japan, ${ }^{30}$ the cumulative incidences of adverse drug reactions extrapolated by the Kaplan-Meier method were $61.0 \%$ and $67.7 \%$ at weeks 28 and 52, respectively, indicating that more adverse drug reactions developed earlier, although delayed-type adverse drug reactions were not observed.

The percentage of patients who discontinued treatment because of adverse drug reactions and abnormal clinical laboratory values was $12.1 \%$ (44/364 patients) and $0.6 \%$ (2/360 patients), respectively. Adverse drug reactions which led to study discontinuation in at least three patients included ejaculation disorder in 15 patients, diarrhea in four patients, and light-headed feeling in three patients. The percentage of patients whose dose was reduced to $4 \mathrm{mg}$ /day because of adverse drug reactions was $11.8 \%$ (43/364), and dose reduction due to abnormal clinical laboratory test values occurred in $0.3 \%$ of patients $(1 / 360)$. Of these adverse drug reactions, ejaculation disorder disappeared in three of 17 patients, 
dizziness on standing up in eight of 10 patients, thirst in four of six patients, light-headed feeling in four of six patients, and nasal congestion in two of five patients during the administration period. Of 91 patients who had ejaculation disorder, five could not be evaluated for resolution of symptoms due to lack of sexual activity and in two patients who underwent transurethral resection of prostate after completion of treatment, but was confirmed in 73 patients after completion of administration and in 11 patients during administration.

Cardiovascular vital signs were recorded for any evidence of a cardiovascular effect. The systolic blood pressure was $137.5 \pm 18.1 \mathrm{mmHg}$ at baseline, $134.7 \pm 17.9 \mathrm{mmHg}$ at week 28, and $134.6 \pm 18.8 \mathrm{mmHg}$ at week 52. The diastolic blood pressure was $80.1 \pm 11.8 \mathrm{mmHg}$ at baseline, $78.4 \pm 12.0 \mathrm{mmHg}$ at week 28 , and $78.9 \pm 12.1 \mathrm{mmHg}$ at week 52 ; the pulse rate was $72.3 \pm 11.4$ beats $/ \mathrm{min}$ at baseline, $72.8 \pm 11.8$ beats $/ \mathrm{min}$ at week 28 , and $74.3 \pm 12.3$ beats $/ \mathrm{min}$ at week 52, showing only a little change in measurements, and not raising clinical concerns.

These study results demonstrated that silodosin improved urinary function and LUTS associated with BPH starting soon after the first administration, without causing delayed adverse drug reactions or loss of efficacy, and confirmed that efficacy was sustained over the course of the study.

An open-label extension study was performed by Marks et al in the US. ${ }^{31}$ The primary objective of this study was to assess safety. Of the 661 participants, $435(65.8 \%)$ completed the study and 431 (65.2\%) experienced 924 adverse events. No serious adverse events occurred that the investigators considered to be drug-related. Adverse events reported most often included retrograde ejaculation (20.9\% of patients), diarrhea (4.1\%), and nasopharyngitis (3.6\%). Orthostatic hypotension and dizziness occurred in $2.6 \%$ and $2.9 \%$ of patients, respectively. The percentage of patients with treatment-emergent adverse events, stratified by preceding double-blind treatment (placebo or silodosin), was higher for de novo (previous treatment with placebo $71.5 \%)$ than for continuing silodosin treatment $(58.3 \%)$. More patients receiving de novo (7.5\%) versus continuing treatment $(1.9 \%)$ discontinued study participation because of retrograde ejaculation. The mean \pm SD IPSS change from baseline (after 12 weeks of previous double-blind therapy) to week 40 (observed cases) was $-4.5 \pm 6.7$ for de novo treatment $(P<0.0001)$ and $-1.6 \pm 6.0$ for continuing treatment $(P<0.01)$. Silodosin was well tolerated and in particular was associated with low incidences of dizziness and orthostatic hypotension. During this extension study, no cardiac disorders and no prolongation of corrected QT interval were found with long-term use of silodosin. Another study ${ }^{42}$ also showed that co-administration of silodosin and phosphodiesterase-5 inhibitors (sildenafil or tadalafil) in healthy men caused no important orthostatic changes in blood pressure or heart rate.

Intraoperative floppy iris syndrome is a complication of cataract surgery observed in patients who have been previously treated with $\alpha_{1}$-blockers, mainly tamsulosin. The clinical manifestations of intraoperative floppy iris syndrome are pupil constriction, fluttering, and billowing of the iris stroma, with a propensity of the iris to prolapse during cataract surgery. ${ }^{42}$ A prospective study was conducted in 1968 Japanese patients receiving various $\alpha_{1}$-blockers, including silodosin, before cataract surgery. ${ }^{43}$ The overall incidence of intraoperative floppy iris syndrome was $1.1 \%$ and, interestingly, no intraoperative cases occurred in patients receiving silodosin. However, one case of intraoperative floppy iris syndrome has been reported in a nine-month, open-label, tolerability study of silodosin. ${ }^{44}$ It is recommended that patients with BPH/LUTS being considered for cataract surgery be questioned to ascertain whether they have taken $\alpha_{1}$-adrenergic receptor antagonists. If so, the ophthalmologist should be prepared for possible modifications to their surgical technique should intraoperative floppy iris syndrome be observed during the procedure.

\section{Conclusion}

$\alpha_{1}$-Adrenergic receptor antagonists remain a mainstay in the treatment of male LUTS and clinical BPH. Silodosin, a new $\alpha_{1 \mathrm{~A}}$-adrenergic receptor antagonist, is now used worldwide. Clinical studies have shown that this selective $\alpha_{1 \mathrm{~A}}$-adrenergic receptor antagonist is very attractive and more effective than placebo for both voiding and storage symptoms, as well as improving measures of quality of life in LUTS arising from BPH. Silodosin has excellent early efficacy, and is at least as effective as other $\alpha_{1}$-blockers. Silodosin shows a strong effect not only on symptoms but also on obstruction as measured by pressure flow studies, a finding perhaps explained by its strong selectivity for the $\alpha_{1 \mathrm{~A}}$-adrenergic receptor. Although, silodosin is a very attractive drug, further studies for efficacy and safety, especially, a long-term study comparing this drug with other $\alpha_{1}$-adrenergic receptor antagonists, are needed.

\section{Disclosure}

MY is a consultant for Kissei Pharmaceutical Co, Astellas Pharma Inc and Pfizer; and is Speaker Honorarium for 
Kissei Pharmaceutical Co, Astellas Pharma Inc, Pfizer, Ono Pharmaceutical Co and Kyorin Pharmaceutical Co. YH is a consultant for Kissei Pharmaceutical Co, Astellas Pharma Inc, Ono Pharmaceutical Co and Pfizer; and is Speaker Honorarium for Kissei Pharmaceutical Co, Astellas Pharma Inc, Pfizer, Kyorin Pharmaceutical Co and Asahi Kasei Pharmaceutical Co. All other authors report no conflicts of interest.

\section{References}

1. American Urological Association Guideline: Management of benign prostatic hyperplasia (BPH). 2010. Chapter 1: Guideline on the management of benign prostatic hyperplasia (BPH). Available from: http://www. auanet.org/content/guidelines-and-quality-care/clinical-guidelines. cfm?sub=bph. Accessed May 13, 2011.

2. Wasserman NF. Benign prostatic hyperplasia: A review and ultrasound classification. Radiol Clin North Am. 2006:44:689-710.

3. Thorpe A, Neal D. Benign prostatic hyperplasia. Lancet. 2003;361: 1359-1367.

4. Roehrborn CG, McConnell JD. Benign prostatic hyperplasia: Etiology, pathophysiology, epidemiology, and natural history. In: Wein AJ, Kavoussi LR, Novick AC, et al, editors. Campbell-Walsh Urology. 9th ed. Philadelphia, PA: WB Saunders; 2007.

5. Emberton M, Cornel EB, Bassi PF, et al. Benign prostatic hyperplasia as a progressive disease: A guide to the risk factors and options for medical management. Int J Clin Pract. 2008;62:1076-1086.

6. Fine SR, Ginsberg P. Alpha-adrenergic receptor antagonists in older patients with benign prostatic hyperplasia: Issues and potential complications. J Am Osteopath Assoc. 2008;108:333-337.

7. Beduschi MC, Beduschi R, Oesterling JE. Alpha-blockade therapy for benign prostatic hyperplasia: From a nonselective to a more selective alpha1A-adrenergic antagonist. Urology. 1998;51:861-872.

8. O'Leary MP. Lower urinary tract symptoms/benign prostatic hyperplasia: Maintaining symptom control and reducing complications. Urology. 2003;62 Suppl 1:15-23.

9. Edwards JL. Diagnosis and management of benign prostatic hyperplasia. Am Fam Physician. 2008;77:1403-1410.

10. Schwinn DA, Roehrborn CG. Alpha1-adrenoceptor subtypes and lower urinary tract symptoms. Int J Urol. 2008;15:193-199.

11. Hieble JP, Bylund DB, Clarke DE, et al. International Union of Pharmacology. X. Recommendation for nomenclature of alpha 1-adrenoceptors: consensus update. Pharmacol Rev. 1995;47: 267-270.

12. Schwinn DA, Johnston GI, Page SO, et al. Cloning and pharmacological characterization of human alpha-1 adrenergic receptors: Sequence corrections and direct comparison with other species homologues. J Pharmacol Exp Ther. 1995;272:134-142.

13. Hawrylyshyn KA, Michelotti GA, Coge F, et al. Update on human alpha1-adrenoceptor subtype signaling and genomic organization. Trends Pharmacol Sci. 2004;25:449-455.

14. Price DT, Lefkowitz RJ, Caron MG, et al. Localization of mRNA for three distinct alpha 1-adrenergic receptor subtypes in human tissues: Implications for human alpha-adrenergic physiology. Mol Pharmacol. 1994;45:171-175.

15. Price DT, Schwinn DA, Lomasney JW, et al. Identification, quantification, and localization of mRNA for three distinct alpha 1 adrenergic receptor subtypes in human prostate. J Urol. 1993;150:546-551.

16. Andersson KE, Lepor H, Wyllie MG. Prostatic alpha 1-adrenoceptors and uroselectivity. Prostate. 1997;30:202-215.

17. Price DT, Schwinn DA, Lomasney JW, Allen LF, Caron MG, Lefkowitz RJ. Identification, quantification, and localization of mRNA for three distinct alpha 1 adrenergic receptor subtypes in human prostate. J Urol. 1993;150:546-551.
18. Nasu K, Moriyama N, Fukasawa R, et al. Quantification and distribution of $\alpha_{1}$-adrenoceptor subtype mRNAs in human proximal urethra. Br J Pharmacol. 1998;123:1289-1293.

19. Rudner XL, Berkowitz DE, Booth JV, et al. Subtype specific regulation of human vascular $\alpha_{1}$-adrenergic receptors by vessel bed and age. Circulation. 1999;100:2336-2343.

20. Souverein PC, Van Staa TP, Egberts ACG, De la Rosette JJMH, Cooper C, Leufkens HG. Use of $\alpha$-blockers and the risk of hip/femur fractures. J Int Med. 2003;254:548-554.

21. Shibata K, Foglar R, Horie K, et al. KMD-3213, a novel, potent, $\alpha_{1 A^{-}}$ adrenoceptor-selective antagonist: characterization using recombinant human $\alpha_{1}$-adrenoceptors and native tissues. Mol Pharmacol. 1955;48: $250-258$.

22. Yamazaki Y. Development of silodosin. Yakugaku Zasshi. 2006;126: 207-208.

23. Tatemichi S, Kobayashi K, Maezawa M, et al. $\alpha_{1}$-Adrenoceptor subtype selectivity and organ specificity of Silodosin (KMD-3213). Yakugaku Zasshi. 2006;126:209-216.

24. Ishiguro M, Futabayashi Y, Ohnuki T, Ahmed M, Muramatsu I, Nagatomo T. Identification of binding site of prazosin, tamsulosin, and KMD-3213 with $\alpha_{1}$-adreneergic receptor subtypes by molecular modeling. Life Sci. 2002;71:2531-2541.

25. Tatemichi S, Kobayashi K, Maruyama I, et al. Effects of silodosin (KMD-3213) on phenylephrine-induced increase in intraurethral pressure and blood pressure in rats - study of the selectivity for lower urinary tract. Yakugaku Zasshi. 2006;126:217-223.

26. Akiyama K, Hora M, Tatemichi S, et al. KMD-3213, a uroselective and long-acting $\alpha_{1 \mathrm{a}}$-adrenoceptor antagonist, tested in a novel rat model. J Pharmacol Exp Ther. 1999;291:81-91.

27. Kawabe K, Yoshida M, Homma Y. Silodosin, a new $\alpha_{1 \mathrm{~A}}$-adrenoceptor selective antagonist for treating benign prostatic hyperplasia: Results of a Phase III randomized, placebo-controlled, double-blind study in Japanese men. BJU Int. 2006;98:1019-1024.

28. Marks LS, Gittelman MC, Hill LA, Volinn W, Hoel G. Rapid efficacy of the highly selective alpha1 A-adrenoceptor antagonist silodosin in men with signs and symptoms of benign prostatic hyperplasia: Pooled results of 2 Phase III studies. J Urol. 2009;181:2634-2640.

29. Chapple CR, Montorsi F, Tammela TJ, Wirth M, Koldewijn E, Fernandez EF; European Silodosin Study Group. Silodosin therapy for lower urinary tract symptoms in men with suspected benign prostatic hyperplasia: Results of an international, randomized, double-blind, placebo- and active-controlled clinical trial performed in Europe. Eur Urol. 2011;59:342-352.

30. Kawabe K, Yoshida M, Arakawa S, Takeuchi H; Silodosin Clinical Study Group. Long-term evaluation of silodosin, a new $\alpha_{1 A^{-}}$ adrenoceptor selective antagonist for the treatment of benign prostatic hyperplasia: Phase III long-term study. Jap J Urol Surg. 2006;19: 153-164.

31. Marks LS, Gittelman MC, Hill LA, Volinn W, Hoel G. Silodosin in the treatment of the signs and symptoms of benign prostatic hyperplasia: A 9-month, open-label extension study. Urology. 2009;74:1318-1322.

32. Yamanishi T, Mizuno T, Tatsumiya K, Watanabe M, Kamai T, Yoshida K. Urodynamic effects of silodosin, a new $\alpha_{1 \mathrm{~A}}$-adrenoceptor selective antagonist, for the treatment of benign prostatic hyperplasia. Neurourol Urodyn. 2010;29:558-562.

33. Matsukawa Y, Gotoh M, Komatsu T, Funahashi Y, Sassa N, Hattori R. Efficacy of silodosin for relieving benign prostatic obstruction: Prospective pressure flow study. $J$ Urol. 2009;182:2831-2835.

34. Miyakita H, Yokoyama E, Onodera Y, et al. Short-term effects of crossover treatment with silodosin and tamsulosin hydrochloride for lower urinary tract symptoms associated with benign prostatic hyperplasia. Int J Urol. 2010;17:869-875.

35. Michel MC. $\alpha_{1}$-Adrenoceptors and ejaculatory function. $B r J$ Pharmacol. 2007;152:289-290.

36. Kobayashi K, Masumori N, Hisasue S, et al. Inhibition of seminal emission is the main cause of an ejaculation induced by a new highly selective $\alpha_{1 \mathrm{~A}}$-blocker in normal volunteers. J Sex Med. 2008;5: 2185-2190. 
37. Schulman CC. Lower urinary tract symptoms/benign prostatic hyperplasia: Minimizing morbidity caused by treatment. Urology. 2003;62 Suppl 3A:24-33.

38. Sanbe A, Tanaka Y, Fujiwara Y, et al. $\alpha_{1}$-Adrenoceptors are required for normal male sexual function. Br J Pharmacol. 2007;152:332-340.

39. Hisasue S, Furuya R, Itoh N, Kobayashi K, Furuya S, Tsukamoto T. Ejaculatory disorder caused by alpha-1adrenoceptor antagonists is not retrograde ejaculation but a loss of seminal emission. Int J Urol. 2006;13: 1311-1316.

40. Roehrborn CG, Kaplan SA, Lepor H, Volinn W. Symptomatic and urodynamic responses in patients with reduced or no seminal emission during silodosin treatment for LUTS and BPH. Prostate Cancer Prostatic Dis. 2011;14:143-148.

41. Homma Y, Kawabe K, Takeda M, Yoshida M. Ejaculation disorder is associated with increased efficacy of silodosin for benign prostatic hyperplasia. Urology. 2010;76:1446-1450.
42. Avisar R, Weinberger D. Intraoperative floppy iris syndrome: Possible relationship with $\alpha_{1}$-adrenergic receptor antagonists. Isr Med Assoc J. 2009;11:42-44.

43. Oshika T, Ohashi Y, Inamura M, et al. Incidence of intraoperative floppy iris syndrome in patients on either systemic or topical $\alpha 1$-adrenoceptor antagonist. Am J Ophthalmol. 2007;143:150-151.

44. United State Food and Drug Administration/Center for Drug Evaluation and Research. Drug Approval Package, RAPAFLO (silodosin) capsules, Application No. 022206. Available from: http:/www.accessdata.fda. gov/drugsatfda_docs/nda/2008/022206s000TOC.cfm. Accessed on April 1, 2011.
Clinical Interventions in Aging

\section{Publish your work in this journal}

Clinical Interventions in Aging is an international, peer-reviewed journal focusing on evidence-based reports on the value or lack thereof of treatments intended to prevent or delay the onset of maladaptive correlates of aging in human beings. This journal is indexed on PubMed Central, MedLine, the American Chemical Society's 'Chemical Abstracts

\section{Dovepress}

Service' (CAS), Scopus and the Elsevier Bibliographic databases. The manuscript management system is completely online and includes a very quick and fair peer-review system, which is all easy to use. Visit http://www.dovepress.com/testimonials.php to read real quotes from published authors.

Submit your manuscript here: http://www.dovepress.com/clinical-interventions-in-aging-journal 